\title{
HIV-TB Treatment Pictogram Tool Designed from Semiotic Analysis for Community Pharmacists in India
}

\author{
J. McDONALD, R. VAILLANCOURT*, P. MISHRA AND ANNIE POULIOT \\ Pharmacy Department, Children's Hospital of Eastern Ontario, 401 Smyth Road, Ottawa K1H 8L1, Ontario, Canada
}

\section{McDonald et al.: HIV-TB Pictograms to Support Pharmacy Counselling}

\begin{abstract}
A pictogram-based counselling pamphlet for pharmacists in India for HIV-TB co-infection and treatment was developed. Pictograms have been shown to improve health literacy and adherence by increasing retention and comprehension of health information, especially in persons with lower levels of literacy. The development of the draft pictogram pamphlet for HIV-TB treatment was based on semiology and adaptation of existing pictograms and incorporation of key counselling points identified in a literature review. A total of 103 articles were reviewed and 21 ( $n=9$ for $\mathrm{HIV}$; $n=12$ for TB) were identified as applicable to pharmacist counselling and were used in the development of key counselling points. Of the 22 pictograms in the draft pamphlet, $n=5$ were redesigned based on existing pictograms, $n=11$ were based on semiotic analysis of existing pictograms, $n=5$ were based on semiology of a web-based image search, and $n=1$ was developed in consultation with Indian pharmacists. The future goal is to validate the pictogram pamphlet as there is a scarcity and oversimplification of validated pictograms targeted to counselling patients with HIV-TB. Although validation of more complicated pictograms in a low-literacy population may be challenging, the aim of pictograms is to enhance recall and deliver the pictogram information in combination with verbal instruction in order to improve adherence and patient outcomes.
\end{abstract}

Key words: HIV, tuberculosis, pictogram, semiology, semiotic analysis

Health literacy, or the ability of a patient to understand medical information and instructions, is an important determinant of medication adherence and the subsequent efficacy and safety of drug therapy ${ }^{[1]}$. Indeed, poor adherence to drug therapy has been associated with adverse drug reactions and hospitalizations ${ }^{[2]}$. Non-adherence to antimicrobial drug therapies for infectious diseases, including tuberculosis (TB) and human immunodeficiency virus (HIV), is of particular concern at both the individual and population level owing to consequent drugresistance and treatment failure ${ }^{[3,4]}$. Adherence to antiretroviral therapy in HIV remains a global concern and it has been demonstrated that low health literacy correlates with poor medication adherence and insight in patients HIV infection ${ }^{[5]}$. Treatment of HIV-TB co-infection represents a particular challenge due to the additive numbers of medications and increased medication regimen complexity. Both polypharmacy ${ }^{[6]}$ and complex regimens involving multiple daily doses and dosing parameters such as food restrictions increase the likelihood of non-adherence ${ }^{[7]}$. In addition, poor knowledge of TB treatment is also a risk factor for treatment non-adherence ${ }^{[8]}$. Therefore, it is imperative

*Address for correspondence

E-mail: rvaillancourt@cheo.on.ca

March-April 2019 that health care professionals deliver novel solutions to overcome literacy-related obstacles to communication in order to optimize patient care.

Although verbal communication is often used to deliver medical information, patients retain more information when verbal instruction is combined with written tools ${ }^{[9]}$. However, in reality, most written drug information is often above the level of comprehension for an average patient ${ }^{[10]}$. Indeed, a systematic review has shown that patients at lower reading levels are, on average, 1.5 to 3 times more likely to experience an adverse health outcome compared to patients at higher reading levels ${ }^{[1]}$. One technique to overcome illiteracy is through the use of pictograms. Pictograms are a graphical or symbolic depiction of an idea, concept or instruction and have been shown to improve retention and comprehension of medical information ${ }^{[11]}$ as well

This is an open access article distributed under the terms of the Creative Commons Attribution-NonCommercial-ShareAlike 3.0 License, which allows others to remix, tweak, and build upon the work non-commercially, as long as the author is credited and the new creations are licensed under the identical terms

Accepted 16 January 2019

Revised 07 May 2018

Received 29 April 2017

Indian J Pharm Sci 2019;81(2):373-379 
as adherence ${ }^{[12]}$, particularly in persons with lower levels of literacy ${ }^{[13]}$. An Indian study evaluating the use of pictograms for patients with HIV found that $32 \%$ of their patients were classified as illiterate and an additional $38 \%$ had only a primary school literacy level ${ }^{[14]}$. Considering that over two-thirds of the HIV population in India may have very limited literacy levels, developing evidence-based counselling tools is of the utmost importance in addressing this public health crisis.

While data specific to HIV-TB is lacking, outpatient pharmacist counselling has been shown to increase patients' medication knowledge and compliance in an Indian setting ${ }^{[15]}$. Specifically, pictograms have been shown to significantly enhance the comprehension of complex medical information in an Indian population. One study, conducted in an outpatient pharmacy in India, included over 200 outpatients with chronic diseases. The knowledge, attitude and practices (KAP) questionnaire was used to assess comprehension of medical information delivered via a validated patient information leaflet containing pictograms. One month after receiving structured counselling with the patient information leaflet, KAP scores were significantly improved in all conditions compared to baseline, with most groups showing mean improvements of 30 points or more in post-education KAP scores ${ }^{[16]}$.

Historically, pictogram development for HIV counselling has been based mostly on expert opinion $^{[14,17,18]}$. As is the standard for most clinical tools, a more objective approach to pictogram development may be accomplished with the use of semiology. Semiology is the formal study of signs and sign-using behaviour. A semiotic analysis involves the systematic study of a group of symbols intending to represent a given idea or concept and identifying both common and differentiating elements. These elements intend to represent the smallest discernible symbolic components that are then combined to form a compound, or a universal theme ${ }^{[19]}$. The results of a semiotic analysis may prove quite helpful in creating methodical instructions for drafting of pictograms by graphic artists.

Based on the aforementioned evidence, the objective of the study was to develop a pictogram-based counselling tool for community pharmacists in India regarding HIV-TB co-infection and its treatment. The first phase of the study was a literature search to identify key counselling points (KCPs) for HIV-TB based on expert review and opinion. The second phase involved a semiotic analysis of the most common imagery for KCPs gathered from the literature. Draft pictograms were designed based on semiology and/or adaptation of existing pictograms. These pictograms were combined with KCPs into a draft counselling pamphlet.

A literature search was conducted in EMBASE, Medline and PubMed to identify articles applicable to pharmacist counselling for HIV-TB. Articles were included without restriction on publication date. A 4-person expert review panel was responsible for evaluating and summarizing the literature, compiling $\mathrm{KCPs}$, and developing an abridged list for use in the counselling tool.

A secondary search of the literature was conducted again in EMBASE, Medline, and PubMed to identify existing pharmaceutical pictograms useful for HIVTB counselling. Documents from organizations such as Féderation Internationale Pharmaceutique (FIP), World Health Organization, and United States Pharmacopeial Convention (USP) were also hand searched for existing pictograms. In some instances where previously validated pictograms were available from FIP, a semiotic analysis was not conducted. These pictograms were re-designed to an Indian context in consultation with practicing Indian pharmacists.

In instances where existing pictograms were unavailable or unsuitable (i.e. less than 2 retrievable pictograms), an internet-based search engine (www. google.com) was used to identify the most common imagery associated with the remaining KCPs. The first 40 returned images were used in the semiotic analysis.

Semiotic analyses were conducted by two independent reviewers to identify both common and differentiating elements in the available imagery. In case of disagreement, a third reviewer was available to resolve any discrepancies.

Following semiotic analysis, a graphic designer was consulted for the design of the pictograms. Pictograms were either re-designed if a suitable FIP pictogram was available or drafted from scratch based on semiotic elements. The design process involved verbal instruction to the artist from the first of two reviewers, review of draft pictograms, and suggestions for redesign by a pharmacist with extensive experience in pictogram development. In addition, Indian pharmacists were consulted with regards to physical (i.e. facial features, hairstyles, clothing) and sociocultural elements (i.e. social dynamics, occupations, hobbies). The 
pictograms were adapted and incorporated into the counselling tool for validation in a subsequent arm of the current study.

A total of 103 articles were reviewed, of which 21 ( $n=9$ for HIV; $n=12$ for TB) were identified as applicable to pharmacist counselling and were used in the development of KCPs. An abbreviated summary of KCPs was chosen for use in the counselling tool and is described in fig. 1. Results of the full literature review and the full list of KCPs are available from the authors upon request.

A semiotic analysis of existing pictograms is found in Table 1. The remaining KCPs, search terms and semiotic analysis are found in Table 2. For simplicity, only results of the semiotic analysis from the first reviewer and only those pictograms chosen for inclusion in the counselling tool are presented.

Previously validated pictograms were available from FIP for four counselling points. Pictograms for nausea, coughing, fatigue and "notify your doctor if you become pregnant" were adapted from the original FIP versions to an Indian context. A suitable pictogram for taking medication every day was found in a similar study that aimed to indicate the importance of taking a full course of antibiotics ${ }^{[20]}$. An internet-based image search was attempted, however the results were non-specific. Therefore, a similar concept (i.e. use of calendar) was used to develop this pictogram without the use of semiology. Owing to their similarity, pictograms 7 and 11 in fig. 1 represent a manipulation of pictogram 2. Semiology was not repeated for pictograms 7 and 11 .

Interestingly, the literature search revealed HIV pictograms $(n=4)$ validated in an Indian population according to internationally accepted criteria ${ }^{[14]}$. Only 4 pictograms met criteria for validation including diarrhoea, vomiting, fever and weight loss. Similar to the FIP pictograms, these pictograms were chosen as the preferred images for adaptation. However, as other pictograms were available in the literature, a semiotic analysis was performed and considered in the graphic redesign, as described in Table 1.

Therefore, of the 22 pictograms in fig. $1, n=5$ were redesigned based on existing pictograms, $n=11$ based on semiotic analysis of existing pictograms, and $n=5$ based on semiology of an internet-based image search. Pictograms $8 \mathrm{a}, 8 \mathrm{~b}$ and $8 \mathrm{c}$ representing the act of taking medications to return to work and enjoy activities, were developed in consultation with Indian pharmacists. The most common occupation was farming with cricket being cited as the most common leisure activity.

Following a search of the literature, it is obvious that pictograms for more general medication counselling (i.e. take with food) are much more common than pictograms for more complicated instructions (i.e. you need to take several medications at the same time). Pictograms for ambiguous instructions (i.e. get help from family and friends) were also scarce with multiple differentiating elements, perhaps owing to their nature and difficulty in accurately representing abstract or intangible emotional actions with pictures ${ }^{[21]}$. The availability of pharmaceutical pictograms in relation to disease-related or sociocultural pictograms may be an artifact of these difficulties.

The decision to adapt existing pictograms to an Indian context was based on previous observations that local versions of pictograms improve comprehension and patients tend to prefer them ${ }^{[20,22]}$.

According to the semiotic analysis of the internetbased image search, there seems to be a powerful symbolism associated with both HIV and TB. In semiology, this is referred to as familiarity or how often the symbol has been encountered, which may be a useful element of sign construction ${ }^{[23]}$. Several common elements were repeated consistently across images, including emaciation for HIV and lungs for $\mathrm{TB}$, which provides a strong rationale for incorporation of these commonalities into pictograms for validation. In keeping with the pattern of images for existing pictograms, identifiable symbols were much less common for non-specific counselling points (i.e. take your medication every day), which is unsurprising as the concepts of HIV and TB have corporeal origins.

As a result, some of the drafted pictograms are more complicated (i.e. take your medications to return to work, if you do not take your medicines your disease will get worse) and validation may prove difficult. Indeed, others have shown that simplicity and concreteness, or the use of real objects, places or people ${ }^{[23]}$, are positively correlated with sign guessability ${ }^{[24]}$. However, the use of simple pictures may be limited to simple instructions. Unfortunately, most HIV-TB counselling points may only be simplified to a certain extent before their meaning is lost. Therefore, complicating factors such as multiple concrete images within a pictogram (i.e. person taking medication superimposed on a calendar) were employed with the hope of improving guessability in exchange for enhancing complexity. Complexity 


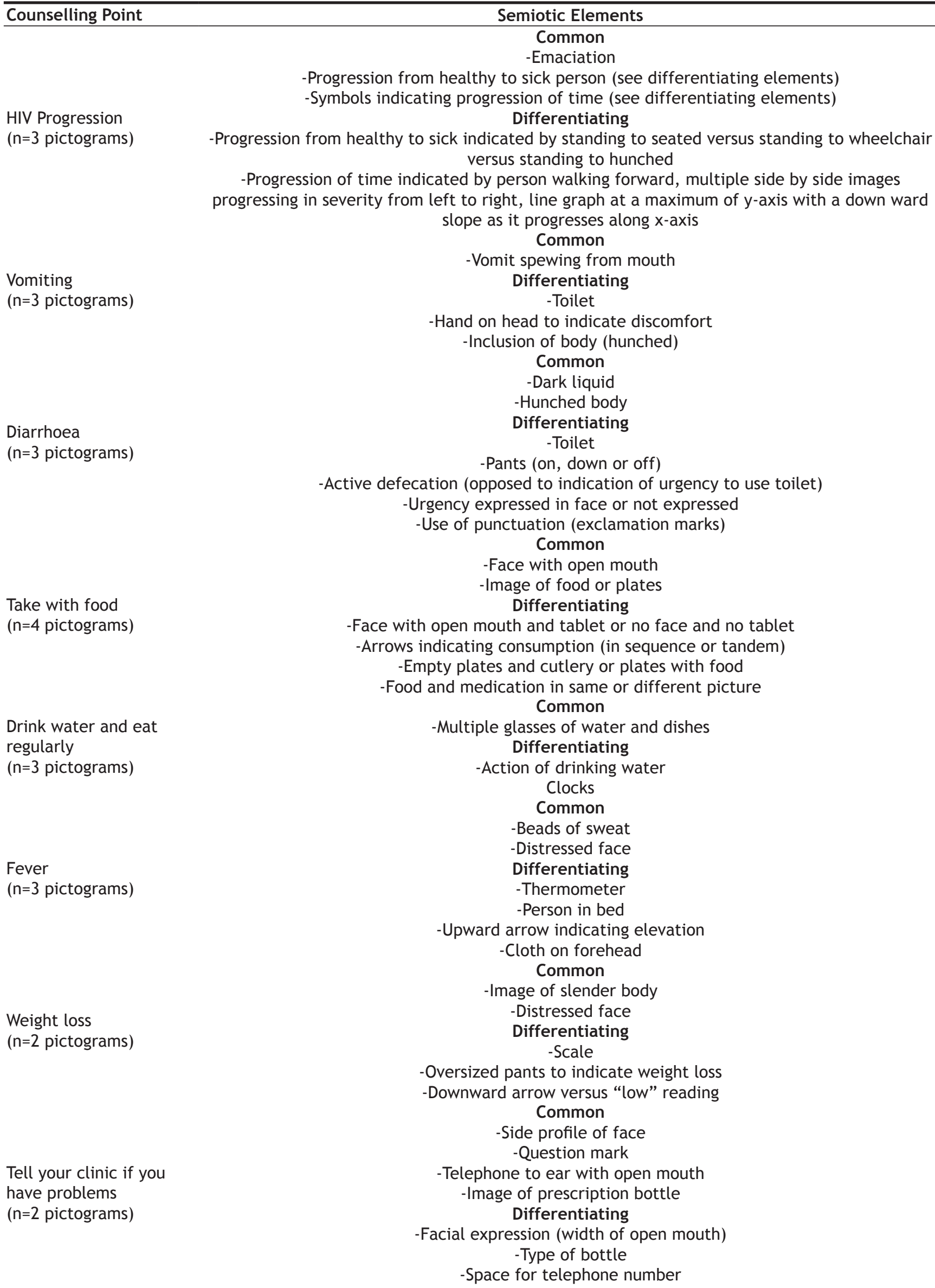

HIV Progression $(\mathrm{n}=3$ pictograms)

Vomiting $(\mathrm{n}=3$ pictograms)

Diarrhoea ( $n=3$ pictograms)

Take with food $(\mathrm{n}=4$ pictograms)

Drink water and eat regularly $(\mathrm{n}=3$ pictograms)

Fever $(\mathrm{n}=3$ pictograms)

Weight loss $(\mathrm{n}=2$ pictograms)

Tell your clinic if you have problems $(\mathrm{n}=2$ pictograms)

Progression from healthy to sick indicated by standing to seated versus standing to wheelchair versus standing to hunched

-Progression of time indicated by person walking forward, multiple side by side images progressing in severity from left to right, line graph at a maximum of $y$-axis with a down ward slope as it progresses along $\mathrm{x}$-axis

Common

-Vomit spewing from mouth Differentiating -Toilet

-Hand on head to indicate discomfort -Inclusion of body (hunched)

\section{Common}

-Dark liquid

-Hunched body

Differentiating

-Toilet

-Pants (on, down or off)

-Active defecation (opposed to indication of urgency to use toilet)

-Urgency expressed in face or not expressed

-Use of punctuation (exclamation marks)

\section{Common}

-Face with open mouth

-Image of food or plates

Differentiating

-Face with open mouth and tablet or no face and no tablet -Arrows indicating consumption (in sequence or tandem)

-Empty plates and cutlery or plates with food

-Food and medication in same or different picture

$$
\text { Common }
$$

-Multiple glasses of water and dishes

Differentiating

-Action of drinking water

Clocks

Common

-Beads of sweat

-Distressed face

Differentiating

-Thermometer

-Person in bed

-Upward arrow indicating elevation

-Cloth on forehead

Common

-Image of slender body

-Distressed face

Differentiating

-Scale

-Oversized pants to indicate weight loss

-Downward arrow versus "low" reading

Common

-Side profile of face

-Question mark

-Telephone to ear with open mouth

-Image of prescription bottle

Differentiating

-Facial expression (width of open mouth)

-Type of bottle

-Space for telephone number 
Do not share your

medications

$(\mathrm{n}=4$ pictograms)

Get help from family and friends ( $n=2$ pictograms)
Common

-Image of prescription

-Hand reaching for medication

-Indication of "no"

Differentiating

-Prescription vial or bag

-Full body image or hands only

-Age of recipient (baby, child, adult)

-Indication of "no" ("x" or raised palm)

Common

-Man and woman (husband and wife)

Differentiating

-Hand gestures versus concept of talking

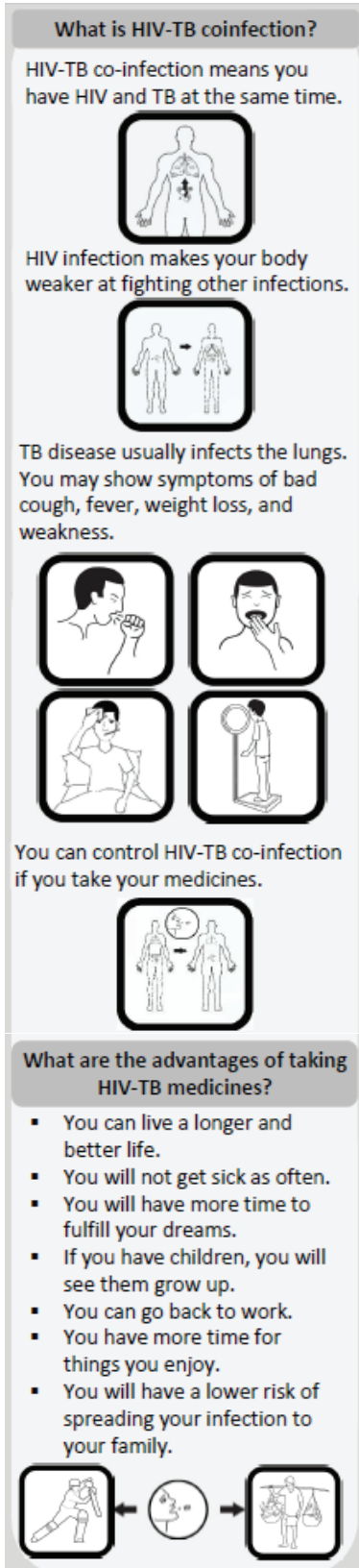

\section{Therapy for HIV-TB Coinfection}

Patient information

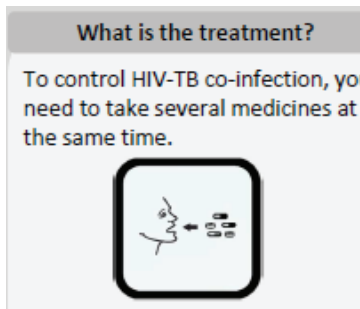

You need to take TB medicines fo many months and HIV medicines for the rest of your life.

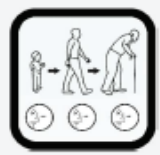

These medicines will stop the growth of HIV and TB bugs and help you become stronger.

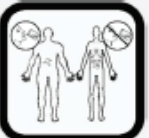

You must continue to take these medicines even when you feel better or have no symptoms.

\section{What are the side effects of HIV-TB medicines?}

Side effects are unpleasant effects that may appear when taking your medicines.

- If you have nausea or vomiting, you should take the medicines with food.

- If you have diarrhea, drink water and eat regularly.

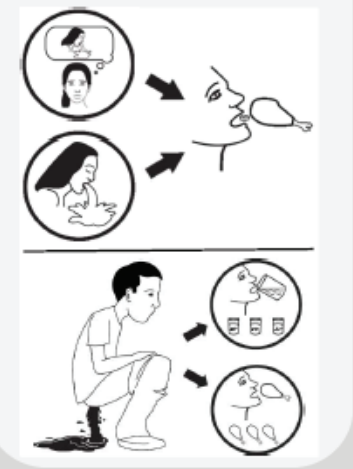

\section{Why is it so important to keep taking your medicines?}

If you do not take your medicines every day, you could make the HIV and TB bugs stronger.

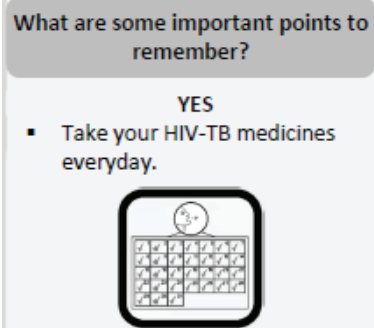

- If you have any problems, tell your clinic.

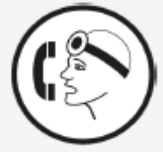

- Develop support and motivation from your family, friends, neighbors, and coworkers.

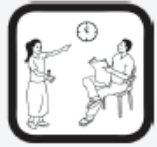

- If you get pregnant while you are taking your medicine, tell the clinic.

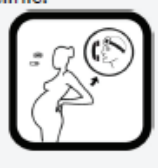

NO

- Do not stop taking your medicines.

- Do not share your medicines with your spouse, partners, or children.

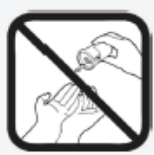

- If you forget a dose, do not take a double dose.

Fig. 1: Summary of key counselling points chosen for the HIV-TB infection counselling tool 


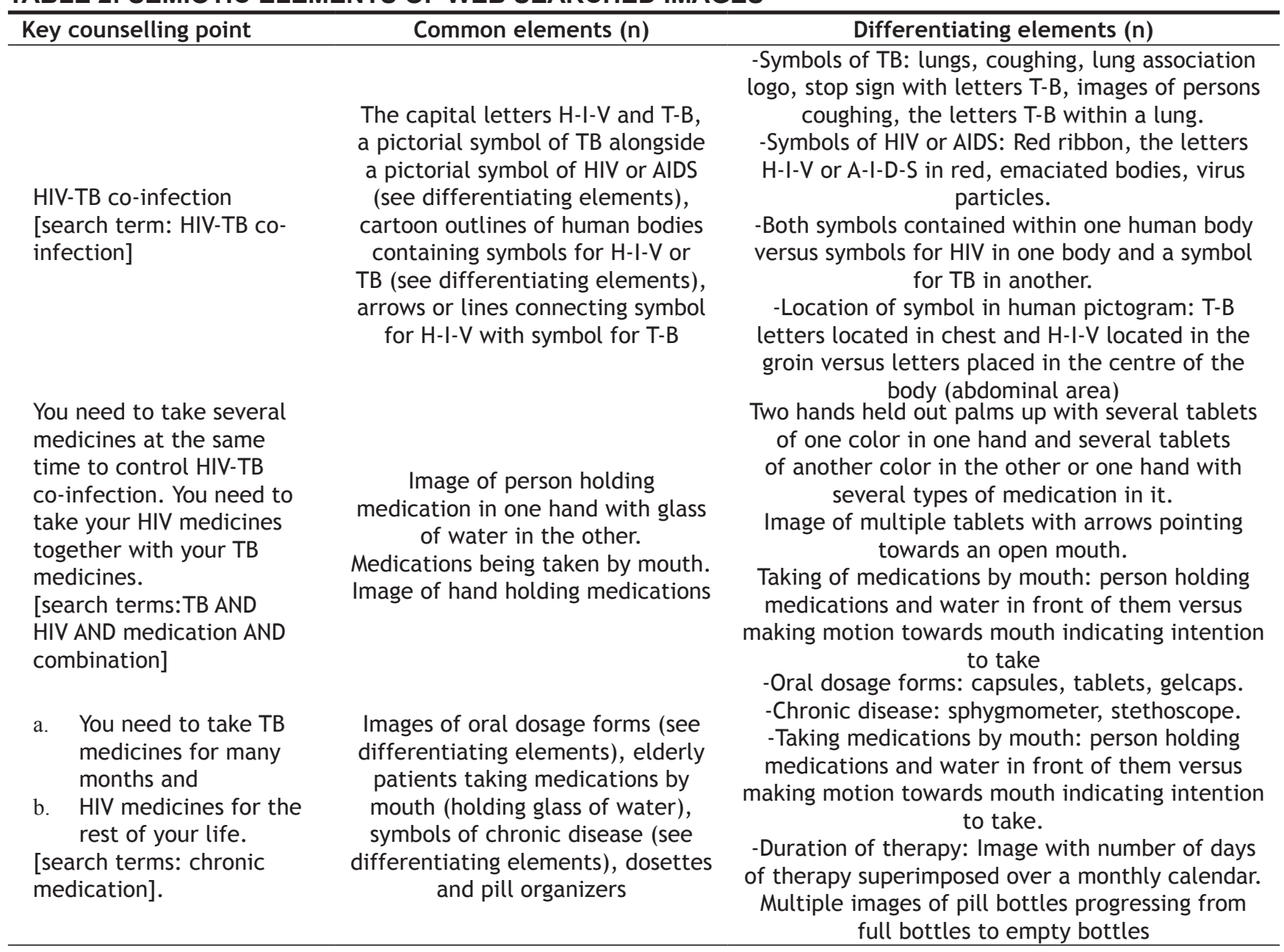

HIV: human immunodeficiency virus; TB: tuberculosis; AIDS: acquired immunodeficiency syndrome; $\mathrm{n}=40$

may increase sign efficacy, but at the expense of increased comprehension time ${ }^{[21]}$. The usefulness of this approach will be further clarified after validation. Ultimately, it is important to remember is that when these images are delivered in combination with verbal and written instruction, the intent is to enhance recall of information rather than achieve perfect guessability. Others have shown this to be a modestly effective strategy to enhance comprehension and recall, although the effects will likely deteriorate over time ${ }^{[11,21]}$.

Perhaps the most important revelation of the current study was the paucity of validated pictograms targeted to counselling patients with HIV-TB. This is particularly alarming as the HIV-TB population is generally of a lower literacy level, increasing their risk of misinterpreting verbal or written health information.

Limitations of the current design may unfortunately delay validation. However, testing and re-design based on review feedback has been shown to increase sign efficacy $^{[25]}$. It is likely that these limitations would be reduced according to patient preference and feedback.
Limitations in the current study that have been shown to decrease sign comprehension include the use of frames and multiple overlapping frames, a cross or " $x$ " to indicate "do not do," and the use of angular lines to indicate movement or pain, or in this instance fever ${ }^{[26]}$. Hence it was chosen to incorporate these elements into the current design as these are prevalent in previously validated pictograms from FIP and USP ${ }^{[27,28]}$.

In conclusion, draft pictograms were successfully developed for use by the community pharmacists in HIV-TB counselling. From our literature searches, it is clear that a larger availability of validated pictograms for targeted counselling of HIV-TB co-infection and its treatment is needed. The currently available pictograms, while useful, are likely be too simple to meet educational requirements. Unfortunately, validating more complicated pictograms in a lowliteracy population may prove difficult, particularly owing to the complicated nature of the instructions to be given. It is expected that simpler pictograms (i.e. for symptoms of TB) will be validated more readily than 
more complex pictograms. Although validation of more complicated pictograms in a low-literacy population could be challenging, the aim of pictograms in general is to enhance recall and deliver them in combination with verbal instruction in order to improve adherence and patient outcomes. Our future goal is to validate these pictograms in the targeted population and assess comprehension and recall of instructions delivered verbally in combination with the counselling tool.

\section{REFERENCES}

1. DeWalt DA, Berkman ND, Sheridan S, Lohr KN, Pignone MP. Literacy and health outcomes: a systematic review of the literature. J Gen Intern Med 2004;19(12):1228-39.

2. Col N, Fanale JE, Kronholm P. The role of medication noncompliance and adverse drug reactions in hospitalizations of the elderly. Arch Intern Med 1990;150(4):841-5.

3. Shin SS, Keshavjee S, Gelmanova IY, Atwood S, Franke MF, Mishustin SP, et al. Development of extensively drug-resistant tuberculosis during multidrug-resistant tuberculosis treatment. Am J Respir Crit Care Med 2010;182(3):426-32.

4. Parienti JJ, Massari V, Descamps D, Vabret A, Bouvet E, Larouzé $\mathrm{B}$, et al. Predictors of virologic failure and resistance in HIV-infected patients treated with nevirapine or efavirenzbased antiretroviral therapy. Clin Infect Dis 2004;38(9):1311-6.

5. Nachega JB, Morroni C, Zuniga JM, Schechter M, Rockstroh $\mathrm{J}$, Solomon S, et al. HIV treatment adherence, patient health literacy, and health care provider-patient communication: results from the 2010 AIDS Treatment for Life International Survey. J Int Assoc Physicians AIDS Care 2012;11(2):128-33.

6. Marcum ZA, Gellad WF. Medication adherence to multidrug regimens. Clin Geriatr Med 2012;28(2):287-300.

7. Stone VE, Hogan JW, Schuman P, Rompalo AM, Howard AA, Korkontzelou $\mathrm{C}$, et al. Antiretroviral regimen complexity, self-reported adherence, and HIV patients' understanding of their regimens: survey of women in the her study. J Acquir Immune Defic Syndr 2001;28(2):124-31.

8. Castelnuovo B. A review of compliance to anti tuberculosis treatment and risk factors for defaulting treatment in Sub Saharan Africa. Afr Health Sci 2010;10(4):320-4.

9. Morris LA, Halperin JA. Effects of written drug information on patient knowledge and compliance: a literature review. Am J Public Health 1979;69(1):47-52.

10. Wolf MS, Davis TC, Shrank W, Rapp DN, Bass PF, Connor $\mathrm{UM}$, et al. To err is human: patient misinterpretations of prescription drug label instructions. Patient Educ Couns 2007;67:293-300.

11. Houts PS, Bachrach R, Witmer JT, Tringali CA, Bucher JA, Localio RA. Using pictographs to enhance recall of spoken medical instructions. Patient Educ Couns 1998;35:83-8.

12. Dowse R, Ehlers M. Medicine labels incorporating pictograms: do they influence understanding and adherence? Patient Educ
Couns 2005;58(1):63-70.

13. Houts PS, Witmer JT, Egeth HE, Loscalzo MJ, Zabora JR. Using pictographs to enhance recall of spoken medical instructions II. Patient Educ Couns 2001;43:231-42.

14. Rajesh R, Vidyasagar S, Varma DM, Sharma S. Design and evaluation of pictograms for communicating information about adverse drug reactions to antiretroviral therapy in Indian human immunodeficiency virus positive patients. J Pharm Biomed Sci 2012;16(10):1-11.

15. Ponnusankar S, Surulivelrajan M, Anandamoorthy N, Suresh B. Assessment of impact of medication counseling on patients' medication knowledge and compliance in an outpatient clinic in South India. Patient Educ Couns 2004;54(1);55-60.

16. Adepu R, Swamy MK. Development and evaluation of patient information leaflets (PIL) usefulness. Indian J Pharm Sci 2012;74(2):174-8.

17. Dowse R, Ramela T, Browne SH. An illustrated leaflet containing antiretroviral information targeted for low-literate readers: development and evaluation. Patient Educ Couns 2011;85(3):508-15.

18. Dowse R, Ramela T, Barford KL, Browne S. Developing visual images for communicating information about antiretroviral side effect to a low-literate population. Afr J AIDS Res 2010;9(3):213-24.

19. Kolers PA. Some formal characteristics of pictograms. Am Sci 1969;57(3):348-63.

20. Dowse R, Ehlers MS. The evaluation of pharmaceutical pictograms in a low-literate South African population. Patient Educ Couns 2001;45(2):87-99.

21. Montagne M. Pharmaceutical pictograms: A model for development and testing for comprehension and utility. Res Social Adm Pharm 2013;9(5):609-20.

22. Goel G. A comparative study to evaluate patients interpretation of U.S.P and locally designed pharmaceutical pictograms. Pharm Times 2010;42(6):16-9.

23. McDougall SJP, Curry BM, de Bruijn O. Measuring symbol and icon characteristics: Norms for concreteness, meaningfulness, familiarity, and semantic distance for 239 symbols. Behav Res Methods Instrum Comput 1999;31(3):487-519.

24. Chan AH, Chan KW. Effects of prospective-user factors and sign design features on guessability of pharmaceutical pictograms. Patient Educ Couns 2013;90(2):268-75.

25. Ringseis EL, Caird JK. The comprehensibility and legibility of twenty pharmaceutical warning pictograms. Proceed Hum Factor Ergon Soc 1995;39(15):974-8.

26. iso.org [Internet].Geneva: International Organization for Standardization [cited 2013 May]. Available from: https:// www.iso.org/standards.html.

27. United States Pharmacopeia dispensing information. Vol. II. Rockville (MD): United States Pharmacopeial Convention Inc.; 1989.

28. fip.org [Internet]. The Hague: International Pharmaceutical federation [cited 2013 May]. Available from: http://www.fip. org/pictograms. 